\title{
Morphogenetic Events in Hair Follicle Heterotypic Spheroids
}

\author{
Ekaterina Kalabusheva, Ekaterina Vorotelyak
}

\section{Laboratory of Cell Biology, Koltzov Institute of Developmental Biology of Russian Academy of Sciences, Moscow, Russian Federation.}

Hair follicles $(H F)$ reconstruction in vitro is a promising field in alopecia treatment and human HF development research. Here, we combined postnatal human dermal papilla (DP) cells and skin epidermal keratinocytes (KCs) in a hanging drop culture to develop an artificial HF germ. The method is based on DP cells hair inducing properties and KCs self-organization. We evaluated two protocols of aggregate assembling. We examined aggregates assembled using DP cells and KCs suspensions (mixed aggregates) and DPCs spheroids covered by KCs (coated aggregates). Mixed HF germ-like structures demonstrated the initiation of epithelial-mesenchymal interaction, including WNT pathway activation and expression of follicular markers.
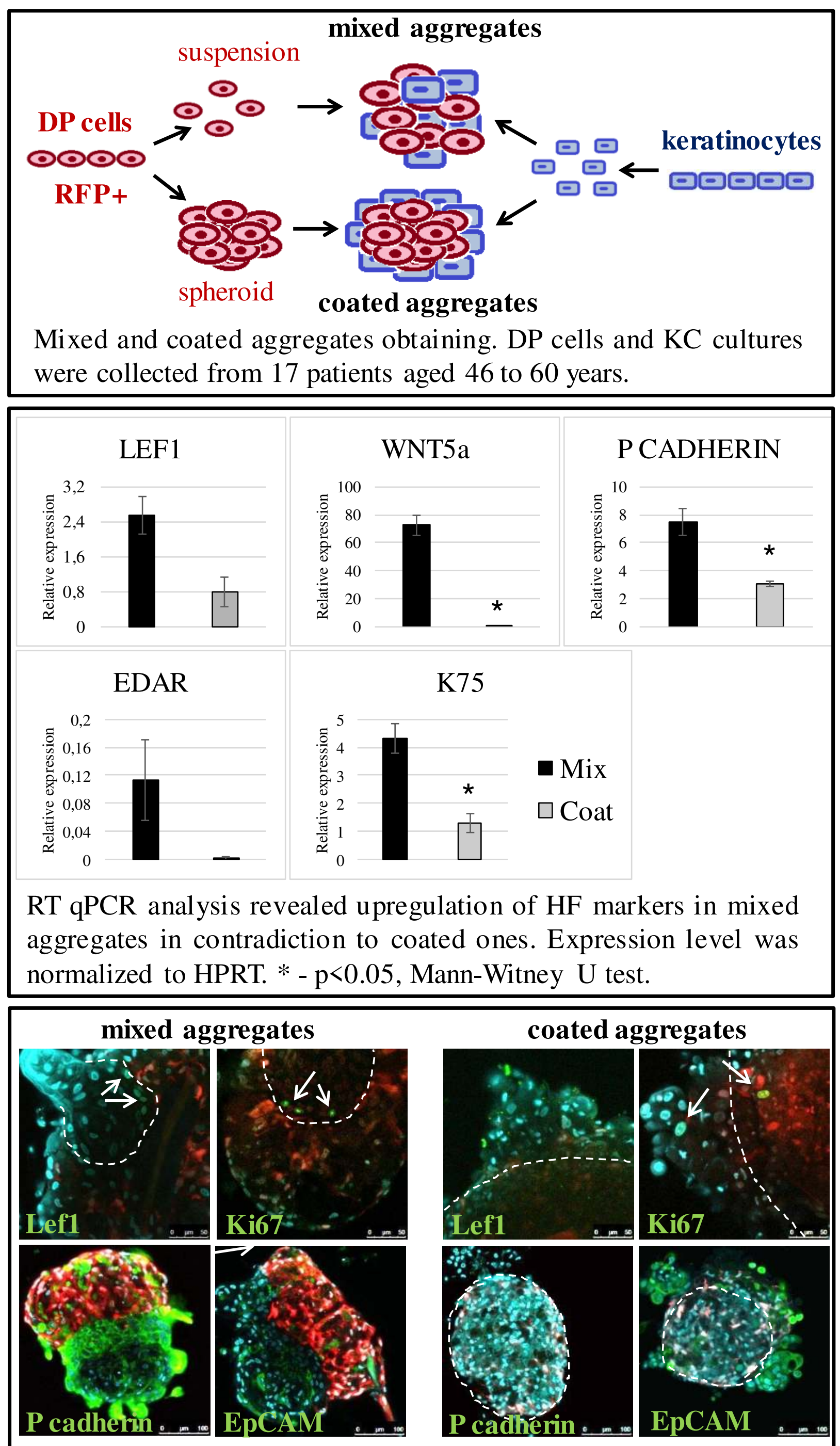

In mixed aggregates DP cells interacted with each other and produced spheroid structures while $\mathrm{KCs}$ tended to form extended trabeculae. Folliculogenesis markers (P cadherin, EpCAM, Lef1) and proliferation marker Ki67 expression patterns correspond to placode stage of HF development. Any morphogenetic events had not been observed in coated aggregates.

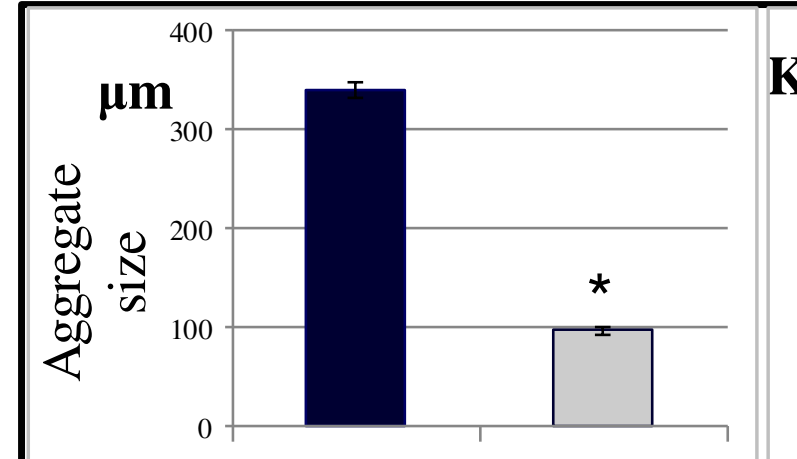

1 passage 12 passage

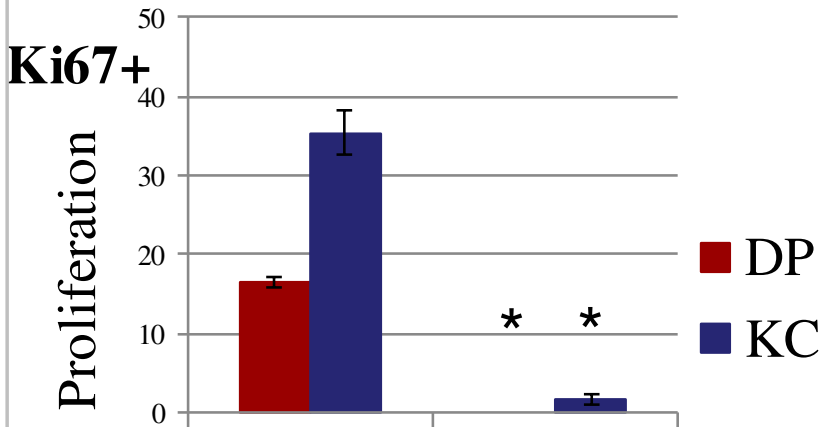

1 passage 12 passage

Downregulation of DPCs hair inducing abilities trough the passaging decreased the size of mixed aggregates and the number of proliferative cells within. Aggregates containing DP cells from the first passage were three times bigger than those made of cells from passage $12{ }^{*}$ - $\mathrm{p}<0.05$, Mann-Witney U test.
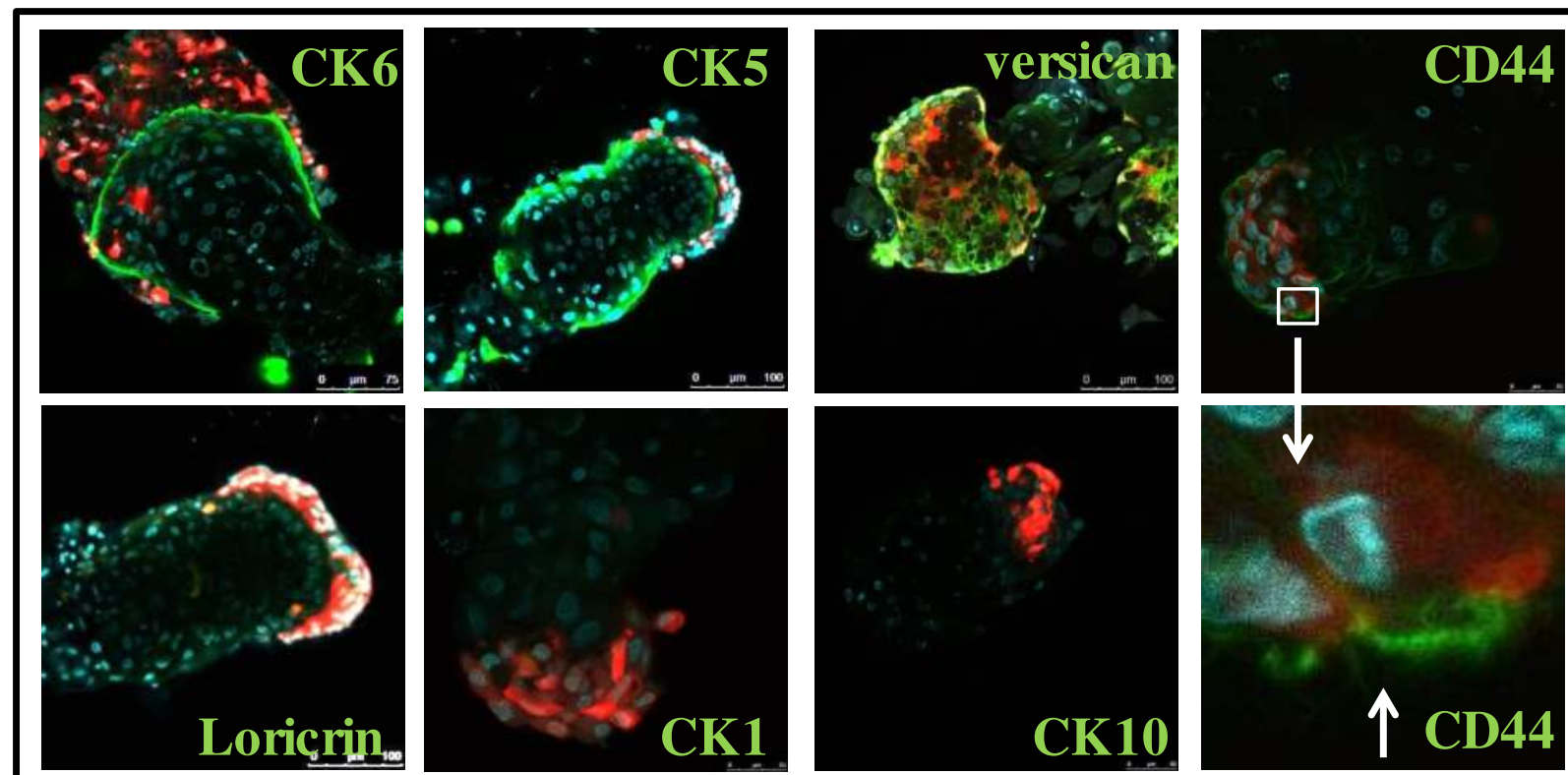

Mixed aggregates structure and morphology had been investigated. Versican positive DP cells formed round-shaped structures; KCs generated multilayered trabeculae embedded into DPCs spheroids CK5 and CK6 positive KCs were found in an external layer of trabeculae, surrounding the epithelial compartment and adjacent to DPCs. We did not found any markers of epidermal differentiation including loricrin, CK 1, and CK10. DPCs and KCs were positive for CD44, hyaluronic acid (HA) receptor. CD44 staining detected microvillus-type plasma membrane protrusions on the DPCs in mixed aggregates.

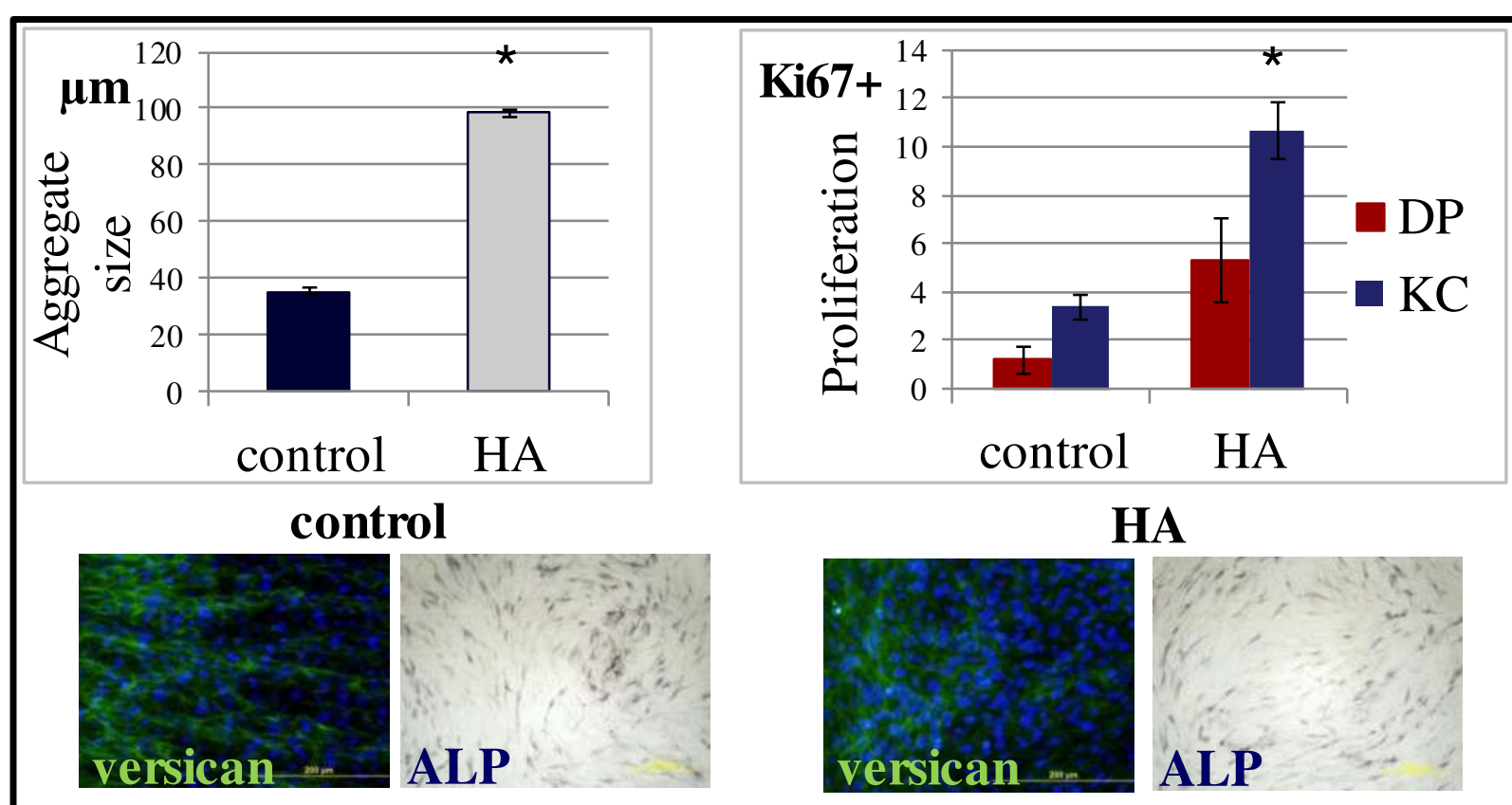

Addition of $0.5 \mathrm{mg} / \mathrm{ml} \mathrm{HA}$ to cell suspension prior to aggregate formation increased the size of aggregates and the number of proliferative cells. However HA did not maintain DPCs specific markers during 2D cultivation (versican and ALP). Therefore, HA influences size of aggregates not by upregulation of DPCs hair inducing abilities. * $-\mathrm{p}<0.05$, Mann-Witney U test.

The long-term goal of our investigation is to develop easily obtainable model system that simulates human HF germ in culture, which could be useful for HF biology research and for clinical applications, providing the suitable system for drug testing and prospective field in $\mathrm{HF}$ reconstruction. The hair germ-like organoid developed in this study allows the search for niche factors maintaining HF morphogenesis.

The work was funded by the Russian Science Foundation 